\title{
ArcheoSciences
}

Revue d'archéométrie

39 | 2015

Varia

\section{Maurice Picon, le GMPCA et l'archéométrie}

\section{Albert Hesse}

\section{OpenEdition}

\section{Journals}

\section{Édition électronique}

URL : https://journals.openedition.org/archeosciences/4542

DOI : $10.4000 /$ archeosciences. 4542

ISBN : 978-2-7535-4778-0

ISSN : 2104-3728

\section{Éditeur}

Presses universitaires de Rennes

\section{Édition imprimée}

Date de publication : 31 décembre 2015

Pagination : 217-218

ISBN : 978-2-7535-4776-6

ISSN : 1960-1360

\section{Référence électronique}

Albert Hesse, « Maurice Picon, le GMPCA et l'archéométrie », ArcheoSciences [En ligne], 39 | 2015, mis en ligne le 31 décembre 2015, consulté le 22 juillet 2021. URL : http://journals.openedition.org/ archeosciences/4542; DOI : https://doi.org/10.4000/archeosciences.4542 


\title{
In memoriam
}

\section{Maurice Picon, le GMPCA et l'archéométrie}

\author{
Albert Hesse ${ }^{a}$
}

En 1968, on ne parlait pas d'archéométrie en France. Tout au plus connaissait-on le terme anglais archaeometry qui avait été créé vers la fin des années 1950 par M. J. Aitken (Hesse, 1987 et 1990). La matière de la discipline n'était cependant pas inconnue puisque, dès 1952, A. Laming-Emperaire publiait La découverte du passé (Laming-Emperaire, 1952) où elle faisait le point sur les méthodes scientifiques qui pouvaient intervenir dans l'analyse et l'interprétation des vestiges archéologiques. Des réunions internationales avaient également lieu régulièrement à Oxford, réunissant, toujours à l'initiative de M.J. Aitken dans le cadre de son laboratoire, des spécialistes et quelques archéologues concernés. La présente note n'a pas l'intention de retracer exhaustivement cette histoire dont on trouvera la substance, entre autres, dans les quelques articles cités en bibliographie, mais seulement de rappeler la contribution de Maurice Picon en la matière.

Jeune chercheur à l'époque et ressentant le manque de contacts avec d'autres personnes impliquées dans le dialogue interdisciplinaire très spécifique Sciences/Archéologie, j’avais pensé que l'animation intellectuelle rénovatrice consécutive aux événements de mai 1968 pouvait être favorable à l'émergence d'une entreprise de réflexion, en groupe, sur la spécificité de nos relations avec les archéologues. J'ai alors entrepris (fin 1968 ou début 1969) de rendre visite aux quelques rares personnes, de statut extrêmement différent, mais véritablement actives dans le domaine. Je n'en ai trouvé que trois qui toutes m'ont accueilli fort courtoisement mais en témoignant d'un niveau d'intérêt très variable pour l'entreprise : Madame Delibrias réalisait au CEA des datations C14 pour les préhistoriens mais l'aspect archéologique ne m'a pas semblé alors faire partie de sa principale préoccupation; J.-C. Gardin, lui-même

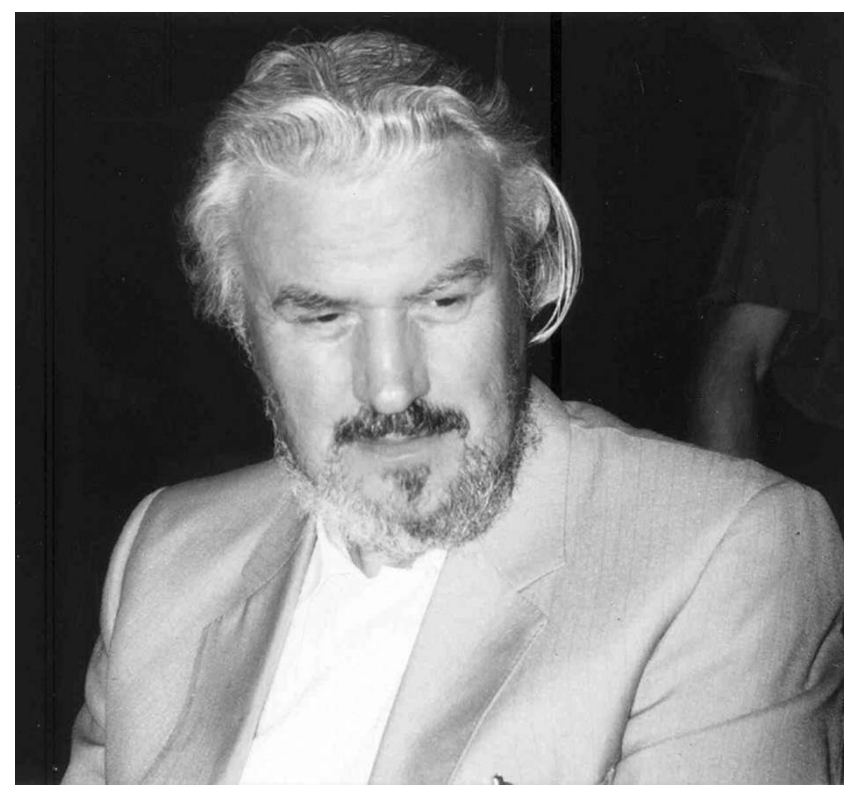

Maurice Picon au colloque de la Société française d'étude de la céramique antique en Gaule (SFECAG) de Mandeure-Mathay, 1990 (photo Y. Rigoir).

archéologue actif au Proche-Orient, et entouré d'un groupe intéressé comme lui, à l'aube du développement de l'informatique, aux processus d'analyse des données, ne m'a pas non plus paru éprouver le besoin de formalisation de ces contacts. Ce n'est qu'avec Maurice Picon, qui avait déjà beaucoup de relations avec un grand nombre d'archéologues intéressés par ce qu'il disait sur les productions céramiques à travers l'analyse de leur composition, que j'ai trouvé alors une oreille attentive à mon initiative de rapprochement. Il y avait évidemment à l'époque, quelques autres personnes,

${ }^{a}$ Ancien Directeur de Recherches CNRS et Université Pierre et Marie Curie Paris 6. 
comme E. Thellier par exemple, que je connaissais bien mais dont je savais, malgré toute l'estime que je leur portais, qu'elles ne seraient pas partie prenante dans ce que j'envisageais, confusément d'ailleurs, de proposer. Ces visites n'ont pas eu de suite immédiate concrète mais les symposiums Archaeometry ont continué de se tenir régulièrement en Angleterre et de prendre de l'importance aux yeux de la communauté scientifique. Jusqu'en 1975, A. Tabbagh, qui m'avait rejoint au Centre de Recherches Géophysiques de Garchy fin 1969, et moi y avons participé, lui surtout, assez régulièrement. En 1976, la première tenue de cette réunion hors d'Angleterre, à Édimbourg en Ecosse, marquait un commencement d'internationalisation. C'est le moment où certaines personnalités européennes, sous la férule de J. Soustelle, ont aussi commencé à se manifester pour créer, en 1975, le PACT (Groupe européen d'études pour les techniques physiques, chimiques et mathématiques appliquées à l'archéologie). Cette création a fait l'objet d'une présentation, pour le moins animée, au symposium d'Edimbourg auquel je n'étais pas présent mais dont les archéomètres français présents sont revenus très remontés en raison de la très modeste compétence de ses promoteurs dans le domaine envisagé. C'est ce groupe de chercheurs, informel au départ, qui a sonné le rassemblement de ceux qui furent les membres fondateurs du GMPCA: J.-N. Barrandon, J. Briard, J. Evin, M. Frizot, L. Langouët, S. Renimel, A. Tabbagh, moi-même et bien sûr Maurice Picon. Si nous lui avons demandé de nous rejoindre bien qu'il ne fût pas participant assidu du symposium Archaeometry, c'est évidemment en raison de l'accueil qu'il m'avait réservé en 1969 et de l'audience dont il jouissait auprès des archéologues. Nous lui devons beaucoup d'avoir accepté la présidence, assez formelle au demeurant, de l'association que nous voulions créer : le G.M.P.C.A. qui signifiait alors Groupe des Méthodes Physiques et Chimiques de l'Archéologie. J'assurais, pour ma part, la charge, assez prenante, de secrétaire général et L. Langouët celle non moins lourde de trésorier.

La suite est assez bien connue (Hesse, 1987 et Tabbagh, 1994) et n'a pas besoin d'être racontée de nouveau. Ces organismes ont continué et progressé pour le plus grand bien de nos disciplines. Maurice Picon a bien soutenu l'équipe pendant plusieurs années à la tête du GMPCA qui a alors organisé son propre colloque en France et fondé la Revue d'Archéométrie.

Le GMPCA, tel que nous l'avons créé avec Maurice Picon, a contribué fortement à l'audience de l'Archéométrie, non seulement en France mais aussi à l'étranger puisque son cadre m'a permis, en 1980 d'inviter pour la première fois le symposium international Archaeometry à Paris et par voie de conséquence de représenter, avec Loïc Langouët, notre pays dans le Standing Committee de cette instance.

Je ne saurais clore cette brève évocation de Maurice Picon sans rappeler deux souvenirs personnels de collaboration scientifique et amicale partagée avec lui. Ce fut tout d'abord à la fin des années 1980, l'emprunt à notre laboratoire d'un magnétomètre pour trouver avec J.-Y. Empereur un magnifique four de potier sur le site de Datça-Resadiye en Turquie. Pas vraiment motivé pour la prospection géophysique, Maurice est retourné à sa céramique, mais m'a ainsi ouvert la voie pour une étude plus complète du site qui s'est avérée être, de par sa complexité, un exemple très significatif de la subtilité avec laquelle il faut quelquefois interpréter la prospection magnétique (Empereur, Hesse et Tuna, 1999). Plus tard, en 1995, c'est à Madagascar que nos missions nous avaient fait, à nouveau, nous rencontrer fortuitement. Cela m'a valu une invitation à l'accompagner dans une passionnante excursion de la journée pour examiner un village de potiers isolé dans les hautes collines des environs d'Antanarivo. Ce fut même pour moi l'occasion de cerner, par une rapide prospection électro-magnétique (EM15-Geonics) le contour d'une activité métallurgique sous-jacente au village et donc antérieure à l'artisanat potier qu’il étudiait (inédit).

\section{Bibliographie}

Hesse A., 1987. Le point sur les réunions d'Archéométrie. Histoire et Mesure, II, (2), p. 131-132.

Hesse A., 1990. Call it Archaeometry. In Mélanges J. Perrot, vol. 1, Paris, Éd. Recherches sur les Civilisations, p. 317-319.

Laming-Emperaire A., 1952. La découverte du passé, Paris, Picard

TABbagh A., 1994. Notes pour servir à l'Histoire du GMPCA. Histoire et Mesure, IX, (3/4), p. 404-408. 